\title{
Are Male Couples Changing Their Sexual Agreements and Behaviors During the COVID-19 Pandemic?
}

\author{
Alison R. Walsh ${ }^{1,2}$ (D) Stephen Sullivan ${ }^{1} \cdot$ Rob Stephenson ${ }^{1,2}$ \\ Accepted: 31 March 2021 / Published online: 15 April 2021 \\ ○) The Author(s), under exclusive licence to Springer Science+Business Media, LLC, part of Springer Nature 2021
}

\begin{abstract}
Sexual agreements are an important element of HIV prevention for many partnered gay, bisexual, and other men who have sex with men (GBMSM). This study describes sexual agreement and sexual behavior changes during the 2020 pandemic among a sample of 215 coupled US GBMSM. Overall, reported behavior shifted towards monogamy. Fifteen percent of respondents developed/ended/changed their agreement during the pandemic; the pandemic factored into 85\% of reported changes. Individuals reported fewer outside sexual partners compared to the 3 months pre-pandemic. More research is needed to investigate shifting behavior and associated risk in order to adapt HIV services during the pandemic.
\end{abstract}

\section{Introduction}

The 2020 pandemic caused by the novel coronavirus SAR$\mathrm{CoV}-2$ continues to have significant impacts on life in the United States and globally. In addition to the pandemic's high morbidity and mortality, social distancing and related mitigation strategies have negatively affected access to healthcare services in the United States [1]. Provider closures, delayed care, and stay-at-home orders/social distancing measures have all contributed to decreased access and utilization of in-person services; reduced access and utilization of sexual health and HIV/AIDS services will likely impact HIV transmission rates and individual risk [2]. These effects have been disproportionately experienced by vulnerable populations, particularly sexual minorities such as gay, bisexual, and other men who have sex with men (GBMSM) [3]. Combined, these factors may result in magnified racial and ethnic disparities in HIV burdens, given the disproportionate impact of COVID-19 on communities of color in the US [4]. To reduce the pandemic's broader, long-term effects, we must understand the impact that the pandemic may have on behavioral HIV risks in order to adapt and/or develop

Alison R. Walsh

walshar@umich.edu

1 Center for Sexuality and Health Disparities, University of Michigan, Ann Arbor, MI, USA

2 Department of Systems, Populations and Leadership, University of Michigan School of Nursing, 400 N Ingalls St., Ann Arbor, MI 48109, USA new programs/services and delivery modes (e.g., telehealth programs) for HIV prevention and care.

In addition to decreases in HIV/AIDS service availability and access, the pandemic appears to be altering behavioral HIV risks. There is growing evidence that intimate relationships, sexual partnerships, and sexual activity frequencies are changing during the pandemic. Mixed-gender studies in southeast Asia and Britain found that the pandemic and "lockdowns" have reduced sex frequency, particularly among women [5, 6]. In contrast, studies with GBMSM conducted early in the pandemic (April-May 2020) in the US had mixed results-Stephenson et al. [7] described increases in sex partners compared to pre-pandemic periods, but with a transition to more condom-protected sex, whereas Sanchez et al. [8] and McKay et al. [9] both documented decreasing numbers of sex partners. However, the pandemic's effects among committed male couples, as well as its impact on sexual behavior further into the pandemic (July-September 2020), are yet unknown. Sexual behavior among coupled GBMSM is uniquely important in HIV/AIDS prevention and research, as transmission between within coupled GBMSM is an important driver of HIV incidence in the US [10]. In addition, GBMSM couples often have explicit sexual agreements that either allow or restrict outside sexual activity, and these agreements have been associated with HIV risk and prevention behaviors [11]. Understanding how the coronavirus pandemic and its associated control measures are impacting GBMSM relationships, sexual agreements, and behavior is a crucial step towards identifying and addressing changing HIV risk in this vulnerable population. This 
study's aim was to describe patterns of changing sexual agreements and behaviors among coupled GBMSM in the United States during the first six months of the coronavirus pandemic, as well as to investigate how the pandemic has motivated for behavioral shifts.

\section{Methods}

Data for the current study was collected from a convenience sample of coupled GBMSM, via online survey from July to September, 2020. Participants were recruited via email from two previous (2017-2018) nationally representative male couples/HIV-related studies-Project Nexus [12], and the Couples Health and Attitudes toward Preexposure Prophylaxis (CHAPS) Study [13]. Ethical approval for the current study was approved by the University of Michigan Institutional Review Board (HUM00182640). Eligible individuals were those who had participated in either of the previously completed studies and also met the current project's eligibility criteria. Project Nexus and CHAPS participants were: 1$) \geq 18$ years; 2) identified as cisgender male; 3 ) in a sexual relationship with a man for $\geq 6$ months (Nexus) or $\geq 3$ months (CHAPS); 4) had internet access. Nexus participants also: 1) had not had an HIV test in the past 3-months; 2) did not report severe intimate partner violence (IPV) or coercion within the past year; 3) were willing to receive rapid home HIV test kits; 4) self-reported concordant HIV-negative or HIV serodiscordant. CHAPS participants also must have reported condomless anal sex with their primary partner within the last 3 months. Previous-study participants (Nexus $\mathrm{N}=666$; CHAPS $\mathrm{N}=799$ ) were emailed invitations to an eligibility questionnaire which inquired about their relationship status. If an email recipient reported they were no longer in a relationship with the partner with whom they had participated in the previous study, they were asked if they were in a relationship with a different man ( $\geq 6$ months duration). If so, the respondent could provide this new partner's contact information which was used to send an eligibility survey link to the new partner $(\mathrm{N}=15)$. Eligibility criteria for the current study was: 1$) \geq 18$ years; 2) identified as cisgender male; 3 ) internet access; 4) not feeling coerced to participate in the study; and 5) in a sexual relationship with a man for $\geq 6$ months. Eligible respondents were then emailed a link to the survey. All emails and links contained embedded identifiers that were used to link data from partners; identifiers were based on couples linked in the previous studies or created for those with "new" partners. Surveys were sent to all eligible respondents, regardless of their partner's participation. Out of 1479 invitations, 298 (20.2\%) completed the eligibility survey. Of these, 8 (2.7\%) were did not report a male partner/relationship and were ineligible. Among eligible respondents, 223 (76.9\%) completed the current study and 215 (96.4\%) of those provided complete data for this analysis-113 individuals and 51 male partner-dyads (102 individuals). Three study participants were randomly selected to receive $\$ 100$ gift cards as a participation incentive.

\section{Measures and Descriptive Analysis}

The survey collected demographics including age, race/ethnicity, educational attainment, employment status, whether the pandemic had negative impacts on employment (e.g., reduced hours, laid off, furloughed), and if individuals had worked/were working from home during the pandemic. We also collected data on COVID-19 confirmed and probable cases - those with a positive test and/or symptoms consistent with COVID-19 infection (coughing, shortness of breath or difficulty breaking, fever, chills, muscle pain, headache, sore throat, and/or new loss of taste or smell [14]) were considered cases. Individuals also reported health conditions that could increase their risk of severe COVID-19 illness (HIV/ AIDS, $\geq 65$ years, cardiovascular and/or lung conditions, cancer treatment, dialysis, liver disease, severe obesity, smoking/vaping, or other unspecified condition).

Relationship characteristics and sexual agreements, behaviors, and activity, during the pandemic and compared to the pre-pandemic period, were assessed. Marital status and relationship length (years) were collected. Participants reported the number of times in the previous 3 months they had anal intercourse (AI) with their primary partner, as well as whether their AI frequency had increased, stayed the same, or decreased compared to the 3 months before the pandemic (approximately November 2019-February 2020). Individuals reported their outside sexual activity - the numbers of outside sexual partners they had in the 3 months prior to the pandemic and during the pandemic, and the types of online activities they'd engaged in during the two time periods (Had an active profile on any dating apps or websites, such as Grindr, Scruff, or Jack'd; Hooked up with someone you met on a dating apps or websites, such as Grindr, Scruff, or Jack'd; Participating in "camming" (i.e., where you interacted with a cam model via video and/or audio) without your partner; Sent sexually explicit messages or emails to someone other than your partner; Sent nude photos of yourself to someone other than your partner).

Participants reported on their pre-pandemic and current sexual agreements with their partner, and if they had changed or ended their pre-pandemic agreement, or had developed a new agreement, during the pandemic. For each period, participants reported whether they had a sexual agreement with their partner, and if so, if it was monogamous or allowed for outside sexual partners (non-monogamous). Participants who reported developing a new agreement or changing/ ending their pre-pandemic agreement during the pandemic 
additionally reported whether the pandemic was the main reason, a contributing factor, or not a part of their decision to change their sexual agreement. Those who reported that the pandemic was the main reason or a contributing factor to their agreement change were then asked to select the way(s) in which the pandemic influenced their decision (To reduce the risk of contracting or transmitting COVID-19; The pandemic made it harder or impossible to meet and/or see other partners; My partner and I saw each other less due to the COVID-19 pandemic; The pandemic increased our commitment to each other and our relationship; and/or other specified reason(s)). For pre-pandemic and pandemic periods, individuals with reported sexual agreements were also asked if they had broken their agreement during that time, and if so, what activities they engaged in with outside partners when breaking their agreement (in-person sexual contact; directly sent or posted online provocative or sexual text messages, photos, and/or videos; online sexual activity (e.g., "camming"); other specified activity). Individuals who reported breaking their agreements during the pandemic were then asked if the whether the pandemic was the main reason, a contributing factor, or not a part of their decision to break their sexual agreement. If the pandemic contributed to the breakage, participants could then select reason(s) for breaking their agreement (I was unable to have sex with my partner at the time due to social or physical distancing measures; My partner wasn't interested in sex because of the pandemic; I wanted to have sex without putting my partner at risk for COVID-19; I was stressed and anxious because of the pandemic; I was bored; I felt isolated and/or lonely; I was emotionally distant from my partner or my partner was emotionally distant from me; My relationship with my partner was suffering because of the pandemic;

I wanted to "get it out of my system" before beginning social distancing; I wanted to live in the moment because the future is uncertain; and/or other specified reason). For the pre-pandemic and pandemic periods, individuals who reported any in-person and/or online sexual activity (overall or when breaking their sexual agreement) were identified. For all measures, means \pm standard deviations (SD) or frequencies were calculated. We also assessed changes in sexual agreements by comparing individual reported preand during-pandemic agreement types.

\section{Results}

The mean $( \pm \mathrm{SD})$ age of the sample $(\mathrm{N}=215)$ was $35.9 \pm 9.2$ years and the mean reported relationship length was $8.2 \pm 5.4$ years. The majority of respondents had a college degree $(77.7 \%)$, were non-Hispanic White $(78.1 \%)$, married (62.3\%), employed (83.1\%), and had at some point worked at home during the pandemic $(63.7 \%)$. Over a quarter of respondents (26.5\%) reported a negative employment change due to the pandemic. A minority of the sample reported having either a positive coronavirus test or exhibiting symptoms consistent with COVID-19 illness during the pandemic (17.7\%) and $37.7 \%$ of respondents reported at least one condition associated with increased risk of serious COVID-19 illness.

Table 1 presents the distributions of sexual agreements and reported behaviors prior to and during the COVID19 pandemic. In the 3 months prior to survey (during the pandemic), respondents reported they'd had AI with their partner an average of $8.5 \pm 13.3$ times, which reportedly represented a decrease in frequency for a third of respondents and an increase for $12.4 \%$, compared to the 3 months before the pandemic. Prior to the pandemic, $31.6 \%$ of respondents did not have an agreement with their primary partner, $23.3 \%$ had a monogamous agreement, and $45.1 \%$ had agreements that allowed for sexual activity with outside partners. Thirtythree individuals (15.4\%) reported a change in their agreement since the beginning of the pandemic. None of those with monogamous agreements reported changes. Of those without an agreement, 6 developed agreements with their partner-2 monogamous and 4 non-monogamous-during the pandemic. Among the 97 individuals who reported having non-monogamous agreements pre-pandemic, 27 (27.8\%) changed their agreements -5 ended their agreement, 14 changed their agreement to monogamy, and 8 remained nonmonogamous but changed the agreement's terms in some way. Thus, during the pandemic, $31.2 \%$ of respondents did not have an agreement with their partner, $30.7 \%$ agreed to be monogamous, and $38.1 \%$ had non-monogamous agreements. The majority of those who changed their agreements $(\mathrm{N}=28)$ reported that the pandemic was the primary reason or a contributing factor in their decision to change, end, or enter into a new agreement. All but 2 of these individuals reported changing their agreement in order to reduce the spread of COVID-19, and 5 reported that an increased commitment to their relationship contributed to their decision to change their agreement during the pandemic. Agreement breakage was generally low in the sample-only 7 reported breaking their agreement during the pandemic and of those, 3 reported that the pandemic played a role in their behavior. The reasons cited for breakage were related to the pandemic itself (e.g., reducing partner's risk) and the mental health effects of the pandemic (e.g., anxiety, boredom).

The presence and number of outside sexual partnerships, both online and in-person, also changed during the pandemic. The number of surveyed men without any online or in-person sexual contacts outside of their primary partner rose from 103 to 114 . The proportion of respondents with in-person, outside sexual partners was reduced from $34.0 \%$ in the 3 months before the pandemic to $24.7 \%$ during the pandemic. Of the 73 individuals with pre-pandemic outside 
Table 1 Demographic, relationship, and behavioral characteristics of an online sample of coupled Gay, Bisexual and other Men who have Sex with Men (GBMSM) $(n=215)$, collected July-September, 2020, United States. Data are N $(\%)^{\mathrm{a}}$ or mean \pm standard deviation (SD)

Characteristic or behavior

Prior to

COVID-19

pandemic

Anal intercourse (AI) frequency (past 3 months)

$8.5 \pm 13.3$

AI frequency during COVID-19 pandemic

Less frequently than 3 months before COVID-19 pandemic

$69(33.0)$

About the same frequency as 3 months before COVID-19 pandemic

$114(54.6)(54.6)$

More frequently than 3 months before COVID-19 pandemic

$26(12.4)$

Sexual agreement with partner

None

68 (31.6)

$67(31.2)$

Monogamous

$50(23.3)$

$66(30.7)$

Non-monogamous

$97(45.1)$

$82(38.1)$

Changed, ended, or developed sexual agreement during COVID-19 pandemic

$33(15.4)$

COVID-19 pandemic was primary or contributing factor in changing, ending, or developing sexual agreement ${ }^{\mathrm{b}}$

Reason for changing, ending, or developing sexual agreement during COVID-19 pandemic $^{c, d}$

Reduce risk of contracting or transmitting COVID-19

$28(84.9)$

Difficult to meet/see outside partners during pandemic

$10(35.1)$

5 (17.9)

Outside sexual partners/activity ${ }^{d, e}$

In-person

$73(34.0)$

$103(47.9)$

$53(24.7)$

Online

$103(47.9)$

$95(44.2)$

None

Number of outside sexual partners (in-person) ${ }^{\mathrm{f}}$

$3.9 \pm 4.0^{\mathrm{e}}$

114 (53.0)

Broken agreement with partner ${ }^{\mathrm{g}}$

COVID-19 contributed to breaking agreement ${ }^{\mathrm{h}}$

$11(7.5)$

$2.5 \pm 2.1$

3

How pandemic contributed to agreement breakage ${ }^{d, i}$

"I was unable to have sex with my partner at the time due to social or physical distancing measures."

"I wanted to have sex without putting my partner at risk for COVID-19."

"I was stressed and anxious because of the pandemic."

"I was bored."

"I felt isolated and/or lonely."

"I was emotionally distant from my partner or my partner was emotionally distant from me."

SD Standard deviation

${ }^{\text {a }}$ Totals may not sum to sample size $(\mathrm{N}=215)$ due to missing data; \% not reported for $\mathrm{N}<5$

${ }^{\mathrm{b}}$ Among those who reported changing, ending, or entering into a sexual agreement with their partner

${ }^{\mathrm{c}}$ Among those who reported the pandemic was a factor in their agreement change

${ }^{\mathrm{d}}$ Responses/categories not mutually exclusive

${ }^{\mathrm{e}}$ Pre-pandemic recall period: 3 months

${ }^{\mathrm{f}}$ Among those with in-person outside sexual partners

${ }^{g}$ Among those who reported having a sexual agreement with their partner

${ }^{\mathrm{h}}$ Among those who reported breaking agreement during COVID-19 pandemic

${ }^{\mathrm{i}}$ Among those who reported that COVID-19 contributed to agreement breakage

partners, $27(37.0 \%)$ reported no outside sexual partners during the pandemic. Among those with outside partnerships, the average number of partners was lower during the pandemic than in the 3 months prior. Individuals who reported in-person sexual contacts during both periods $(\mathrm{N}=46)$ had average $2.2 \pm 4.1$ fewer partners during the pandemic. The sample's online sexual activity with outside partners shifted less than in-person activity; 8 fewer individuals had online sexual activity during the pandemic than in the 3 months prior. 


\section{Discussion}

The results presented here describe and highlight the ways in which a small sample of coupled GBMSM are changing their sexual relationships and activities in response to the first five to seven months of the coronavirus pandemic. Although the majority of respondents reported that they had not changed their AI frequency, sexual agreement, number and/or presence of outside sexual partners, and/ or online sexual activity during the pandemic, a notable portion of the analyzed sample did report changes. A third of respondents reported lower AI frequency with their primary partner than in the pre-pandemic period. Among those who had changed their sexual behavior outside of their main partnership, we identified a general trend towards monogamy and fewer outside sexual contacts and activity, both in-person and to a lesser extent, online. In addition, data from those who reported behavioral changes sheds light on the emotional, protective (and potentially altruistic), and logistical motivations prompting these shifts (e.g., reducing COVID-19 transmission, difficulties meeting partners).

Among those who changed their behavior, there was a material shift towards fewer sexual partners. Thirtythree study participants, $15 \%$ of the sample, had adopted, changed, or ended a sexual agreement with their partner during the pandemic, the majority of whom became explicitly monogamous. Outside of sexual agreements, the proportion of individuals who reported in-person sexual partners other than their primary partner during the pandemic dropped to $25 \%$, from $34 \%$ in the pre-pandemic period, and the average number of outside sexual partners for these sub-samples was also lower. In contrast, early in the pandemic, Stephenson et al. [7] reported that a sample of GBMSM in the US had increased their number of sex partners. However, McKay et al. [9] and Sanchez et al. [8] both reported decreases in sex partners early in the pandemic (April-May 2020). These contradictory results may reflect differences between single and coupled GBMSM, or other within-GBMSM diversity such as age or health conditions, and/or evolving behavior in responses to the changing pandemic. It may be that men in committed partnerships were/are more willing to change their behavior in order to protect their partner, particularly those with conditions that put them at higher risk for serious COVID-19 illness. Stephenson et al. also that the majority of their study population did not agree that reducing sexual partnerships was important during the pandemic, whereas in our sample, the majority of agreement changes were attributed to the pandemic. The specific impetuses for changes were a desire to prevent COVID-19 transmission, logistical issues related to pandemic-related social distancing and restrictions, and increased relationship quality. Although we cannot draw conclusions based on our scant data, these motivations suggest that as the pandemic's trajectory, mitigation strategies, and social/economic environments evolve, behaviors may continue to change in response. It may be that the perceived severity of the pandemic and COVID-19 illness is increasing, and/or social distancing-acceptance is becoming more commonplace as we progress further into the pandemic, resulting in increased willingness to change in-person contact patterns. While our results suggest recently reported behavioral modifications may represent conscious choices in direct response to perceived COVID-19 risk, there is a clear need for continued research in this area.

In addition to reducing outside sexual activity and altering sexual agreements, relationship characteristics appear to be changing for some GBMSM couples during the pandemic. Close to $50 \%$ of the study population reported AI frequency changes and a third reported less AI during the pandemic than in the months prior; this finding again concurs with McKay et al. [9] and Sanchez et al. [8]. Evidence from sexual agreement data also suggest that relationship characteristics in GBMSM couples are mutable during the pandemic. Although the sample of respondents who reported sexual agreement changes was small, $18 \%$ reported that the change was influenced by increased commitment to their partner.

This small study's descriptive results suggest that lifestyle and behavioral adaptations spurred by the pandemic could also be reducing individual HIV/AIDS risk for some coupled GBMSM. However, we did not collect data on condom use or PrEP/PEP and therefore cannot assert congruence with HIV risk. Moreover, this study was limited by its cross-sectional design and sampling method. Behavioral adaptations and relationship changes are likely to continue to change across the pandemic's trajectory and in relation to fluctuating transmission rates and control measures. The surveys were administered approximately 5-7 months into the pandemic, prior to vaccine distribution in the US - these results may not be applicable to future or longer-term trends, particularly post-vaccination. Additionally, the study sample was drawn from previous studies, as opposed to a random sample of the population, and was relatively small, which limits generalizability. This study also may not be generalizable to male couples with sociodemographic and/or relationship characteristics not fully represented in the study's sample-e.g., single GBMSM; those in shorter-term relationships; racial/ethnic minorities. Given the higher COVID19 burden among communities of color in the US, these GBMSM in these populations may have different behavioral and relationship changes in response to COVID-19 risk.

Overall, this study highlights the evolving impact the coronavirus is having on GBMSM relationships and sexual 
behaviors. Although the changes documented in this study's small sample are not necessarily negative or positive, they may represent larger shifts in HIV risk profiles in a vulnerable population. HIV prevention programs and interventions aimed at coupled GBMSM will need to adapt to not only pandemic restrictions, but the changing needs of their target population. Thus, it remains important to continue to investigate GBMSM behaviors and relationships in order to develop and implement effective, evidence-based HIVprevention and related services.

Author Contributions All authors contributed to the study conception, design, material preparation, data collection. Analysis and the first draft of the manuscript were completed by A. Walsh. All authors commented on previous versions of the manuscript, and read and approved the final manuscript.

Funding This work is supported by the National Child Health and Human Development of the National Institutes of Health under award number R01HD078131. The content is solely the responsibility of the authors and does not necessarily represent the official views of the National Institutes of Health.

\section{Declarations}

Conflict of interest The authors have no relevant financial or non-financial interests to disclose.

Ethical Approval The authors assert that all procedures contributing to this work comply with the ethical standards of the relevant national and institutional committees on human experimentation and with the Helsinki Declaration of 1975, as revised in 2008. Ethical approval for the current study was approved by the University of Michigan Institutional Review Board (HUM00182640).

Informed Consent Informed consent was obtained from all individual participants included in the study.

\section{References}

1. Cole B. Health Policy Brief No. 19: The Impact of the COVID-19 Pandemic on Access to Health Care. 2020.

2. Jenness SM, Le Guillou A, Chandra C, Mann LM, Sanchez T, Westreich D, et al. Projected HIV and bacterial STI incidence following COVID-related sexual distancing and clinical service interruption. medRxiv. 2020;2020.09.30.20204529.

3. Tang K, Gaoshan J, Ahonsi B. Sexual and reproductive health (SRH): A key issue in the emergency response to the coronavirus disease (COVID-19) outbreak. Reprod Health. 2020;17(1):1-3.

4. Yancy CW. COVID-19 and African Americans. JAMA. 2020;323(19):1891-2.

5. Arafat SMY, Alradie-Mohamed A, Kar SK, Sharma P, Kabir R. Does COVID-19 pandemic affect sexual behaviour? A cross-sectional, cross-national online survey. Psychiatry Res. 2020;289:113050.

6. Jacob L, Smith L, Butler L, Barnett Y, Grabovac I, McDermott D, et al. Challenges in the Practice of Sexual Medicine in the Time of COVID-19 in the United Kingdom. J Sex Med. 2020;17(7):1229-36.

7. Stephenson R, Chavanduka TMD, Rosso MT, Sullivan SP, Pitter RA, Hunter AS, et al. Sex in the time of COVID-19: results of an online survey of gay, bisexual and other men who have sex with men's experience of sex and HIV prevention during the US COVID-19 epidemic. AIDS Behav. 2020;25(1):40-8.

8. Sanchez TH, Zlotorzynska M, Rai M, Baral SD. Characterizing the impact of COVID-19 on men who have sex with men across the United States in April, 2020. AIDS Behav. 2020;24(7):2024-32.

9. McKay T, Henne J, Gonzales G, Quarles R, Gavulic KA, Garcia Gallegos S. The COVID-19 pandemic and sexual behavior among gay and bisexual men in the United States. SSRN Electron J. 2020

10. Sullivan PS, Salazar L, Buchbinder S, Sanchez TH. Estimating the proportion of HIV transmissions from main sex partners among men who have sex with men in five US cities. AIDS. 2009;23(9):1153-62.

11. Rios-Spicer R, Darbes L, Hoff C, Sullivan PS, Stephenson R. Sexual agreements: a scoping review of measurement, prevalence and links to health outcomes. AIDS Behav. 2019;23(1):259-71.

12. Stephenson R, Freeland R, Sullivan SP, Riley E, Johnson BA, Mitchell J, et al. Home-based HIV testing and counseling for male couples (Project Nexus): a protocol for a randomized controlled trial. JMIR Res Protoc. 2017;6(5):e101.

13. Stephenson R, Chavanduka TM, Sullivan S, Mitchell JW. Correlates of successful enrollment of same-sex male couples into a web-based HIV prevention research study: cross-sectional study. JMIR Public Heal Surveill. 2020;6(1):e15078.

14. Centers for Disease Control and Prevention (CDC). Symptoms of Coronavirus [Internet]. 2020 [cited 2020 Jul 1]. Available from: https://www.cdc.gov/coronavirus/2019-ncov/symptoms-testing/ symptoms.html

Publisher's Note Springer Nature remains neutral with regard to jurisdictional claims in published maps and institutional affiliations. 